\begin{tabular}{ll}
\hline INDEX TO ADVERTISERS & \\
\hline Bruker Nano Surfaces Division & Cover 4 \\
Diatome & Cover 2 \\
EDAX & $\mathrm{A} 8$ \\
Electron Microscopy Sciences, EMS & Cover 3 \\
Panasonic i-Pro & $\mathrm{A} 4$ \\
Tescan & $\mathrm{A} 7$ \\
\hline
\end{tabular}




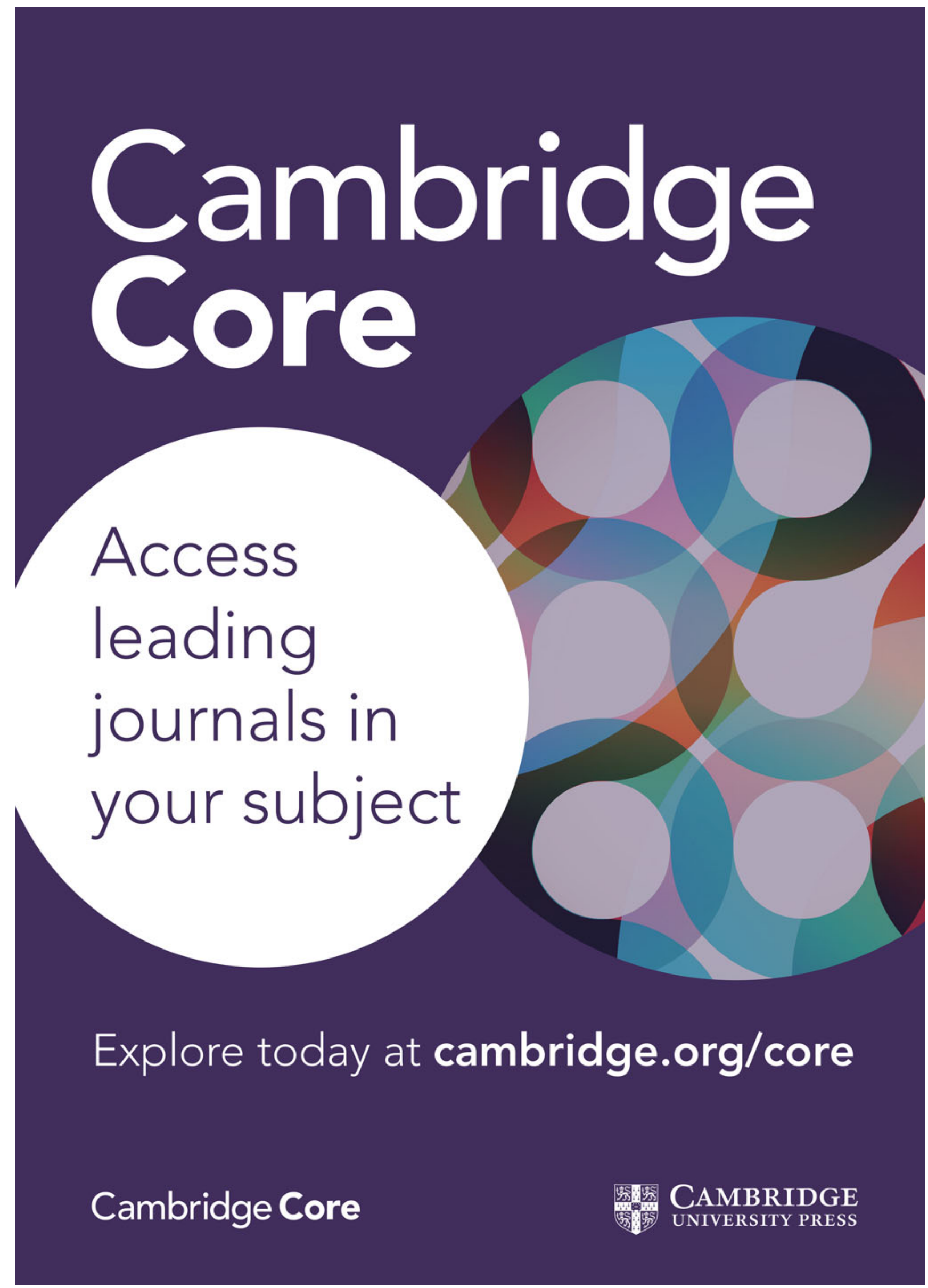




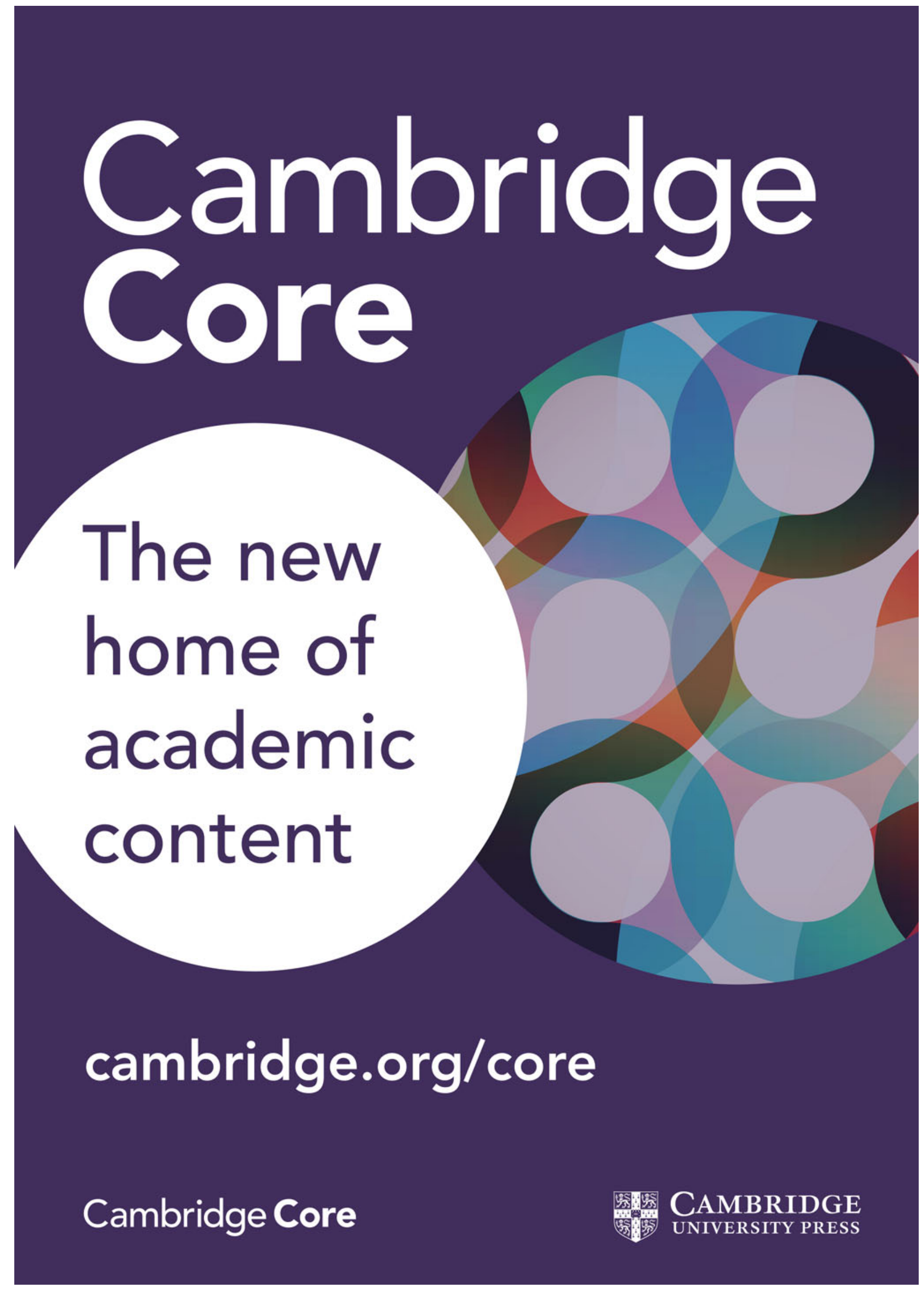


ELECTRON MICROSCOPY SCIENCES...

your source for the latest. the best, the most advanced
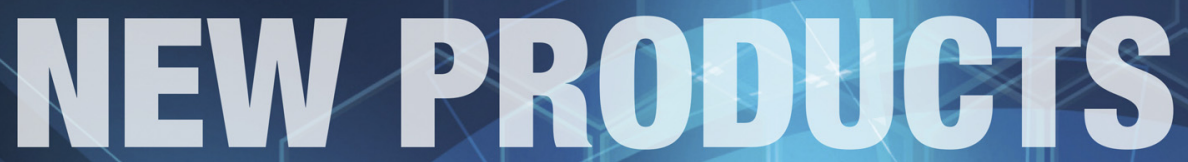

Please visit our website to view or download our NEW PRODUCT FOCUS Catalog; featuring all these products and dozens more.

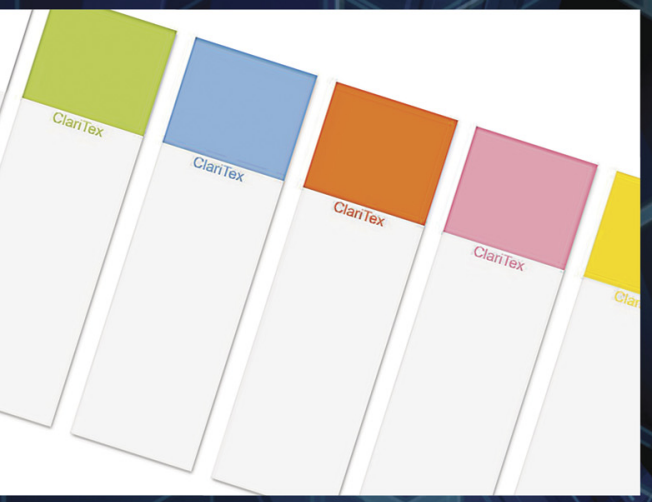

Claritex Slides

Ready-to-use slides are manufactured from super grade glass with uniform thickness, fulfilling optical requirements for light microscopy.

\section{Electron Microscopy Sciences}

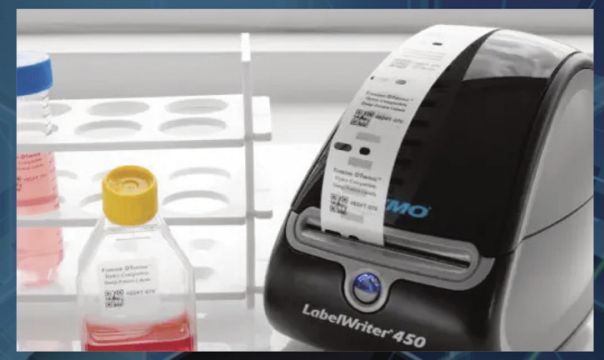

DYMO-Compatible Gryogenic Labels Including CryoSTUCK ${ }^{\circledR}$ Thermal Transfer Labels and Freezer-DTermo ${ }^{\text {TM }}$ Deep Freeze Labels.

\section{CellCeps+ Heated Forceps}

For orientation and positioning of histological specimens during embedding in paraffin wax.

\section{PEN Foil}

Bottom Sterile Dishes Ideal for laser microdissection.
Diamond ${ }^{\circledR}$ Sureflow Bottle Top Dispensers

Precise and easy dispensing of laboratory reagents with an ergonomic design.
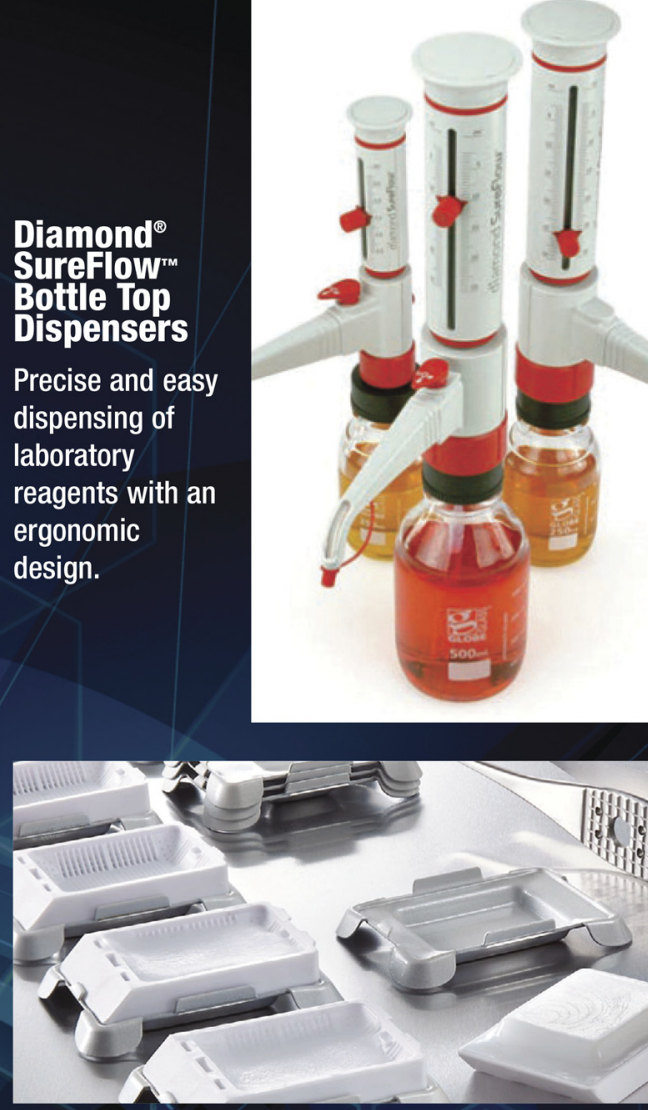

Fast-Cooling Non-Stick Metal Base Molds

Reuseable and disposable molds also available.

Vortex ${ }^{\mathrm{Tm}}$

Biopsy Cassettes

Small and Micro size. Biopsy Paper as well!

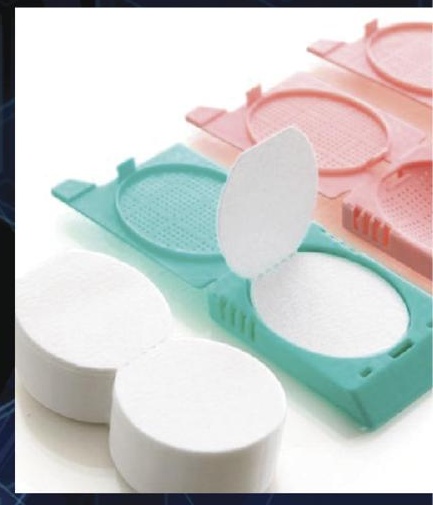

EMS has it!

Scan here to view our NEW PRODUCT FOCUS as a downloadable PDF...

P.0. Box $550 \bullet 1560$ Industry Rd. • Hatfield, PA 19440

Tel: (215) 412-8400 • Fax: (215) 412-8450

email: info@emsdiasum.com or stacie@ems-secure.com

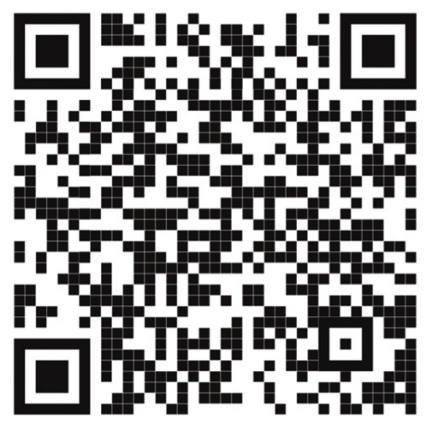




\section{World's Most Advanced Photothermal IR Spectroscopy Solutions}
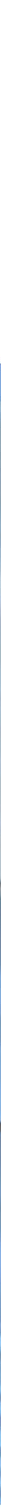

\section{Enable Analytical FT-IR at the Nanoscale}

Bruker's nanoscale infrared (nanolR) spectrometers measure spatially varying physical and chemical properties in a diverse range of fields, including polymers, 2D materials, life science and micro-electronics industry. Featuring proprietary spectroscopy and AFM technology, our instruments have been adopted by leading scientists at top research universities, national laboratories, and major chemical/materials companies worldwide. With an impressive and growing publication record, our customers are proving daily how these systems enable advanced academic discovery and help industrial companies solve critical process problems.

Only Bruker's nanolR solutions provide:

- Rich, interpretable nanolR spectra directly correlated to FTIR

- Hyperspectral AFM-IR for high-resolution, nanolR spectroscopy in seconds
- Sub-10nm resolution chemical imaging with Tapping AFM-IR

- NEW Surface Sensitive nanolR mode for thin films

Take your materials research to the next level today. Visit www.bruker.com/nanolR, email productinfo@bruker.com or call +1.408.376.4040/866.262.4040 for more information today.

Innovation with Integrity 